\title{
Anticancer activity of ursolic acid on retinoblastoma cells determined by bioinformatics analysis and validation
}

\author{
Dan Zhou ${ }^{1}$, Qi Bao ${ }^{1}$, Songbin Fu' ${ }^{2}$ \\ ${ }^{1}$ Department of Ophthalmology, Suzhou High Tech Zone People's Hospital, Suzhou, China; ${ }^{2}$ Department of Ophthalmology, Harbin Medical \\ University, Harbin, China \\ Contributions: (I) Conception and design: D Zhou, Q Bao; (II) Administrative support: S Fu; (III) Provision of study materials or patients: D Zhou, Q \\ Bao; (IV) Collection and assembly of data: D Zhou, Q Bao; (V) Data analysis and interpretation: Q Bao, S Fu; (VI) Manuscript writing: All authors; \\ (VII) Final approval of manuscript: All authors. \\ Correspondence to: Songbin Fu. 157 Baojian Road, Nangang District, Harbin 150076, China. Email: fusongbin_2021@163.com.
}

\begin{abstract}
Background: This article aims to explore whether ursolic acid (UA) inhibits the progression of retinoblastoma $(\mathrm{Rb})$ by regulating stearoyl-CoA desaturase (SCD).

Methods: The Gene Expression Omnibus (GEO) database was used to filter the chip, then the GEO2R software was used to analyze the microarray data (GSE97508, GSE24673, and GSE110811). Gene set enrichment analysis (GSEA) was used to analyze the relationship between the expression level of SCD and the proliferation, migration, invasion, and inflammation in Rb patients. SO-RB50 and Y79 cell proliferation, migration, and invasion were assessed by the CCK-8 assay, the colony formation assay, the Transwell assay, and the wound scratch test. The protein expression levels of SCD were measured by western blot. The mRNA expression levels of IL-8, IL-6, CXCL1, and CCL2 were measured by RT-qPCR. The protein expression levels of IL-8 and IL-6 were measured by ELISA. A xenograft nude mouse model was established to evaluate the effect of UA on tumor growth in male BALB/c mice.

Results: The expression levels of SCD were related to cell proliferation, migration, invasion, and inflammation. UA inhibited SO-RB50 and Y79 cell proliferation, migration, and invasion. At the same time, UA suppressed tumor growth in the xenograft nude mouse model. Overexpression of SCD promoted SORB50 and Y79 cell proliferation, migration, invasion, and inflammation, while SCD knockout inhibited SO-RB50 and Y79 cell proliferation, migration, invasion, and inflammation. Importantly, UA inhibited the proliferation, migration, and invasion of Rb cells through SCD inhibition.

Conclusions: UA inhibited the proliferation, migration, and invasion of Rb cells through SCD. This provides a new scientific basis for targeted therapy of Rb.
\end{abstract}

Keywords: Retinoblastoma (Rb); stearoyl-CoA desaturase (SCD); proliferation; migration; invasion; inflammation

Submitted Aug 17, 2021. Accepted for publication Sep 28, 2021.

doi: $10.21037 /$ atm-21-4617

View this article at: https://dx.doi.org/10.21037/atm-21-4617

\section{Introduction}

Retinoblastoma $(\mathrm{Rb})$ originates from the cells of the embryonic nuclear layer of the retina. Among all malignant tumors in children, the incidence is second only to leukemia, with an annual incidence of $1 / 20,000-1 / 15,000$, and has a genetic predisposition (1). About $95 \%$ of children are younger than 5 years old at the time of onset. About $75 \%$ of children have monocular $\mathrm{Rb}$, and the age of onset is 2 to
3 years. The onset of binocular $\mathrm{Rb}$ is earlier (2-4). There is no gender difference in onset. Globally, there are 9,000 new cases every year (5) and 3,000-4,000 deaths (6). There are about 1,100 new cases every year domestically $(5,7)$. Rb not only harms children's health and visual function, but also threatens the lives of children. In developing countries, most children with $\mathrm{Rb}$ have developed to the late stage when they are found, and the survival rate is much lower than that in 


\section{Page 2 of 16}

developed countries $(8,9)$. Early detection of $\mathrm{Rb}$ can provide better treatment for children, and early diagnosis and early treatment are key to improving the cure rate and reducing the mortality rate (10). Targeted biological therapy is the future direction of research and development. Therefore, indepth study of the related mechanisms of $\mathrm{Rb}$ tumor growth provides a scientific basis for early diagnosis, treatment, and prognosis evaluation of $\mathrm{Rb}$.

Ursolic acid (UA), a pentacyclic triterpenoid of $\alpha$-aromatic alcohol type, is mainly distributed in gardenia, oldenlandia diffusa, bearberry leaves, loquat leaves, Ligustrum lucidum, hawthorn, and Prunella vulgaris. The molecular formula of UA is $\mathrm{C}_{30} \mathrm{H}_{48} \mathrm{O}_{3}$. Studies have shown that UA has a variety of significant pharmacological effects, such as protecting the liver (11), alleviating lipid accumulation (12), anti-tumor effects (13), and antiinflammatory effects (14). Studies have confirmed that UA can inhibit the proliferation of malignant cells, promote their apoptosis, affect the invasion and metastasis of malignant tissues, and inhibit the angiogenesis of malignant cells and epithelial-mesenchymal transition $(15,16)$. UA can inhibit the cell proliferation of $\mathrm{Rb}$ (17), but there is no research showing how UA exerts anticancer effects on Rb.

Stearoyl-CoA desaturase (SCD) is a key enzyme in the process of de novo fatty acid synthesis. SCD belongs to the dehydrogenase family, and its role is to catalyze the conversion of saturated fatty acid (SFA) into monounsaturated fatty acid (MUFA). This is achieved by introducing a cis double bond between carbon 9 and 10 of the acyl-CoA substrate. The most common process is to convert palmitic acid and stearic acid into palmitoleic acid and oleic acid, respectively $(18,19)$. SCD is a protein anchored on the endoplasmic reticulum membrane with a protein size of about $37 \mathrm{kDa}$. Studies on tumor metabolism have shown that lipid synthesis in tumors, especially the production of fatty acids, is increased. The expression of SCD is significantly positively correlated with the occurrence and development of lung cancer, breast cancer, colorectal cancer, kidney cancer, liver cancer, and other tumors (20-24). Through bioinformatics analysis, the expression level of SCD has been shown to be related to the proliferation, migration, invasion, and inflammation of $\mathrm{Rb}$. However, the role of SCD in Rb has not yet been reported.

Therefore, in this study, we aimed to explore the effect of $\mathrm{UA}$ on the growth of $\mathrm{Rb}$ and its mechanism of action. It was predicted by bioinformatics methods that SCD can affect the growth of Rb. We hypothesized that UA could inhibit the proliferation, migration, invasion of $\mathrm{Rb}$ by
Zhou et al. Bioinformatics study of Ursolic acid in retinoblastoma

regulating USD. Firstly, UA was tested in vitro and was shown to inhibit the proliferation, migration, and invasion of SO-RB50 and Y79 cells, and the inhibitory effect of UA on $\mathrm{Rb}$ growth was also assessed in vivo. Then, SCD agonists and inhibitors were transfected into SO-RB50 and Y79 cells. Through in vitro experiments, it was found that SCD promoted the proliferation, migration, and invasion of SORB50 and Y79 cells, and inhibited inflammation. Finally, in vitro experiments verified that UA can down-regulate the expression of SCD, thereby inhibiting the growth of $\mathrm{Rb}$.

We present the following article in accordance with the ARRIVE reporting checklist (available at https://dx.doi. org/10.21037/atm-21-4617).

\section{Methods}

\section{Microarray analysis}

The Gene Expression Omnibus (GEO) database was used to filter the chip, then the GEO2R software (https://www. ncbi.nlm.nih.gov/geo/geo2r/) was used to analyze the microarray data (GSE97508, GSE24673, and GSE110811) $(25,26)$. Gene chips were screened according to the conditions of $\mathrm{P}<0.05$ and $\log \mathrm{FC}$ greater than 1 . In order to obtain more accurate information, the screening conditions of SCD with $\mathrm{P}<0.05$ and $\log \mathrm{FC}$ greater than 1 were improved. The study was conducted in accordance with the Declaration of Helsinki (as revised in 2013).

\section{Gene set enrichment analysis (GSEA)}

GSEA from the TCGA database $(27,28)$ was performed to analyze the relationship between the expression level of SCD and the proliferation, migration, invasion, and inflammation in $\mathrm{Rb}$ patients.

\section{Cell culture}

SO-RB50 and Y79 cells were obtained from American Type Culture Collection. SO-RB50 and Y79 cells were cultured in RPMI-1640 medium with $10 \%$ exosome-free fetal bovine serum (FBS). The cells were subsequently incubated at $37{ }^{\circ} \mathrm{C}$ in a tissue culture chamber with $95 \%$ oxygen $\left(\mathrm{O}_{2}\right)$ and $5 \%$ carbon dioxide $\left(\mathrm{CO}_{2}\right)$.

\section{Cell treatment}

UA was obtained from Mansite (Chengdu, China) with 
a purity of $>98 \%$. Cells were divided into four groups, including the $0 \mu \mathrm{M}$ UA group, $10 \mu \mathrm{M}$ UA group, $20 \mu \mathrm{M}$ UA group, and $240 \mu \mathrm{M}$ UA group.

\section{Cell transfection}

Plasmid cloning DNA (pcDNA), pcDNA-SCD, shRNANC, shRNA-SCD 1\#, and shRNA-SCD 2\# were obtained from GenePharma (Shanghai, China) and were used for efficiently transfection of Rb cells. According to the manufacturer's instructions, the above plasmids diluted by Lipofectamine 3000 (Invitrogen, CA, USA) were transfected into SO-RB50 and Y79 cells, respectively.

\section{Cell counting kit-8 (CCK-8) assay}

Cell proliferation was measured using the CCK-8 assay kit (Dojindo, Japan). A total of $100 \mu \mathrm{L}$ of cell suspension was added to each well of a 96-well plate (Corning, NY, USA). The cells were incubated at $37{ }^{\circ} \mathrm{C}$ and $5 \% \mathrm{CO}_{2}$ for $24 \mathrm{~h}$. Then, $10 \mu \mathrm{L}$ of CCK-8 solution was added to each well (being careful not to create bubbles in the wells when dripping). The cells were placed in an incubator for $1-4 \mathrm{~h}$. The absorbance was measured at $450 \mathrm{~nm}$ with a microplate reader (Thermo Fisher Scientific, USA). The experiment was repeated 3 times.

\section{Colony formation assay}

SO-RB50 and Y79 cells were pretreated with phosphate buffered saline (PBS). The cells were then cultured in a 6-well plate with 500 cells per well for 14 days. After that, the medium was discarded, and the cells were fixed with ethanol for $30 \mathrm{~min}$ and stained with $0.5 \%$ crystal violet. Finally, the cells were rinsed with deionized water to dry, and images were taken for observation.

\section{Transwell assay}

A Matrigel-covered Transwell chamber (24 wells) from BD Biotech was used for the experiment. The Transwell chamber was pre-placed in a 24-well plate, and $600 \mu \mathrm{L}$ of DMEM medium containing $10 \%$ FBS was added to the bottom of it. The resuspended cells $(100 \mu \mathrm{L})$ were seeded into the upper chamber (150,000 cells per well). After incubating for $48 \mathrm{~h}$ in an incubator, the cells were slowly washed twice with $1 \times$ PBS, and were fixed in $4 \%$ paraformaldehyde for $20 \mathrm{~min}$ at room temperature. The cells were stained using $0.1 \%$ crystal violet for $20 \mathrm{~min}$. An optical microscope was employed to capture images. The cells were observed and counted, and the results were depicted in statistical graphs. The experiment was repeated 3 times.

\section{Wound scratch test}

Each group of cells was marked, and a square mark was drawn about $2 \mathrm{~mm}$ wide on the back of a 6-well plate. The cell suspension was placed into a 6 -well plate with $10 \%$ FBS and DMEM medium. After the cells were overgrown, a small pipette tip was used to make a vertical mark on the back of the 6-well plate. Then, the plate was put in a $37^{\circ} \mathrm{C}$, $5 \% \mathrm{CO}_{2}$ incubator for cultivation. The 6-well plate was imaged under an inverted optical microscope (Olympus, Japan) for observation. The images were taken at 0 and $24 \mathrm{~h}$, and the square was used for positioning when imaging. Image J software was employed to measure the width of the scratch area and calculate the healing rate of the cell scratches. The experiment was repeated three times.

\section{ELISA}

To quantify cytokine levels, specific ELISA kits for IL-8 and IL-6 were used according to the manufacturer's instructions. In short, samples from different experimental groups and standard samples of known concentration provided by the kit were added to the kit plate, incubated with the kit reagents, and analyzed by measuring the absorbance at $450 \mathrm{~nm}$. Later, using the standard samples, a standard curve was created with GraphPad Prism 7 software to interpolate the sample absorbance values and obtain the cytokine concentration in each case.

\section{Real-time reverse transcription-polymerase chain reaction (qRT-PCR)}

According to the manufacturer's protocol, total RNA in SO-RB50 and Y79 cells was isolated using the TRIzol reagent kit (Invitrogen, Beijing, China). The MiReasy Mini Spin Column Kit (Qiagen Company, Germany) was used to obtain RNA with higher purity according to the manufacturer's protocol. The NanoDrop 2000 was used to check the concentration of the total RNA immediately, which was then reverse transcribed as soon as possible to avoid degradation. The PrimeScript RT Reagent Kit (TakaRa, Dalian, China) was used for reverse transcription 
to synthesize cDNA. The synthesized cDNA was amplified by PCR using SYBR Premix Ex Taq ${ }^{\mathrm{TM}}$ II (TakaRa, Dalian, China), according to the instructions then the program on the Bio-Rad CFX-96 system was set (Bio-Rad, CA, USA). Glyceraldehyde-3-phosphate dehydrogenase (GAPDH) was used for normalization. The qRT-PCR data were analyzed using the $2^{-\Delta \Delta \mathrm{Ct}}$ method to calculate the relative expression levels of mRNA.

\section{Western blot assay}

Proteins were isolated from SO-RB50 and Y79 cells using RIPA lysis buffer (Beyotime Institute of Biotechnology, Shanghai, China) according to the manufacturer's protocol. The BCA Protein Assay Kit (Beyotime Institute of Biotechnology, Shanghai, China) was employed to detect the protein concentrations. The proteins were separated by $10 \%$ dodecyl sulfate-polyacrylamide gel electrophoresis (SDS-PAGE). Subsequently, proteins were transferred onto polyvinylidene difluoride (PVDF) membranes (Merck Millipore) by electroblotting. In Tris Buffered Saline Tween (TBST), the membranes were blocked with 5\% skim milk for $1 \mathrm{~h}$, and the membranes were incubated with the primary antibody, rabbit anti-SCD, overnight at $4{ }^{\circ} \mathrm{C}$. Membranes were then incubated with corresponding antibodies conjugated to HRP for $1 \mathrm{~h}$ at room temperature. The ECL reagent was used to visualize targeted bands, and quantitative analysis was carried out by image laboratory software (Bio-Rad). $\beta$-actin was used as the internal control to standardize sample loading. The band densities were determined and analyzed with an automatic digital gel image analysis system (Bio-Rad CFX-96, CA, USA).

\section{Nude mouse subcutaneous xenograft model}

A xenograft model was generated as previously described (29). All mice were weighed before the start of the experiment. All animal experiments were carried out according to the NIH Guide for the Care and Use of Laboratory Animals and were approved by the Suzhou high tech Zone People's Hospital. A protocol was prepared before the study without registration. Briefly, 4-week-old athymic balb/c nude male mice weighing $19.56 \pm 2.37 \mathrm{~g}$ were randomized into treatment and control groups $(\mathrm{n}=5)$. SO-RB50 cells were subcutaneously injected $\left(5 \times 10^{6}\right.$ cells/mouse) into each mouse. The control group was treated with $0.1 \%$ DMSO and the treatment group received $200 \mathrm{mg} / \mathrm{kg}$ i.p. UA twice a week (30). The treatment was continued for 7 weeks from the date of randomization.
The tumor sizes were recorded once every week and were determined by a Vernier caliper and calculated. At the end of 7 weeks, mice were sacrificed by a lethal intraperitoneal dose of phenobarbital ( $40 \mathrm{mg} / \mathrm{kg}$ b.w.), and tumor volumes and weight were measured.

\section{Statistical analysis}

Using GraphPad Prism 7.0, an unpaired $t$-test with Welch's correction was performed to assess the endpoints, including differences in SCD expression, proliferation, migration, invasion, and cytokine levels. All statistical tests were twosided, and $\mathrm{P}<0.05$ was considered significant.

\section{Results}

\section{Network pharmacology analysis of in $R b$}

Swiss Target (top 15) and TargetNet (pro $>0$ ) were used to predict the targets of UA. Using the intersection point between SwissTarget and TargetNet, a total of 48 targets of UA were finally obtained. GEO2R was used to analyze the up-regulated genes in the GSE97508, GSE24673, and GSE110811 datasets. Using the intersection point with the UA targets, a common gene SCD is finally obtained (Figure 1A). The results showed that the expression of SCD in the GSE97508, GSE24673, and GSE110811 datasets was significantly higher than that in normal human plasma (Figure 1B). Figure 1C showed that SCD expression was correlated with disease severity in the GSE110811 dataset. Grouped by the median SCD expression level, patients with $\mathrm{Rb}$ in the GSE110811 dataset were divided into 2 groups with high and low SCD expression. GSEA was used to analyze the relationship between SCD expression level and cell proliferation, migration, and invasion. The results showed that the negative regulatory cell proliferation and migration gene sets were significantly enriched in the low SCD expression group, while the cell invasion gene set was enriched in the high SCD expression group (Figure 1D-1F).

\section{$U A$ inbibited the proliferation of $R b$ cells in vitro}

The CCK-8 assay results showed that UA inhibited SORB50 and Y79 cell viability compared with the $0 \mu \mathrm{M}$ UA group (Figure $2 A, 2 B$ ). The results of the clone formation assay showed that UA suppressed SO-RB50 and Y79 cell proliferation compared with the $0 \mu \mathrm{M}$ UA group (Figure 2C). 
A

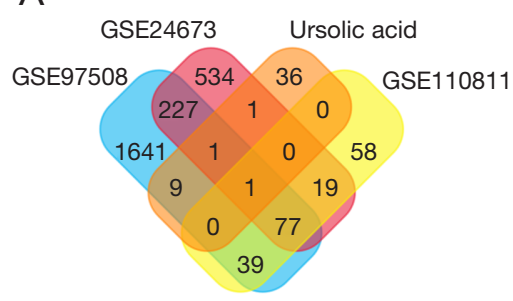

D

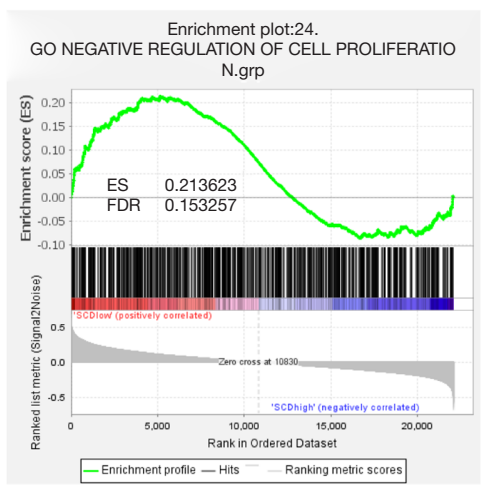

B

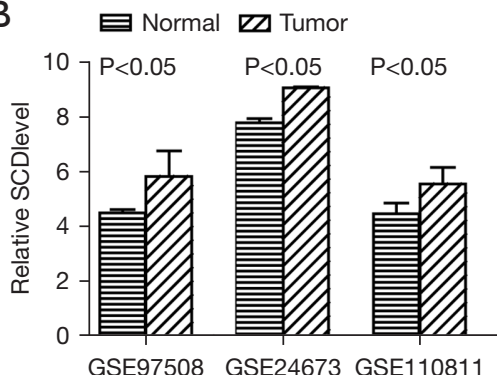

E

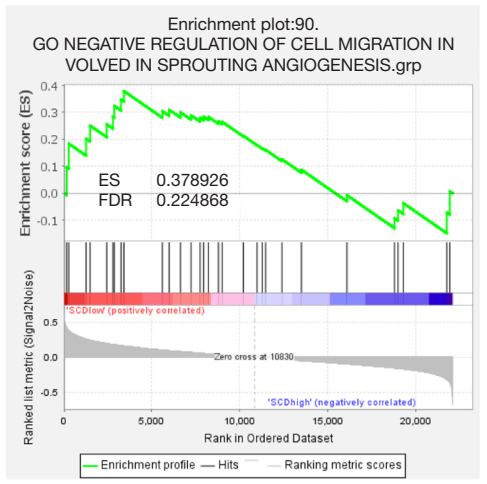

C

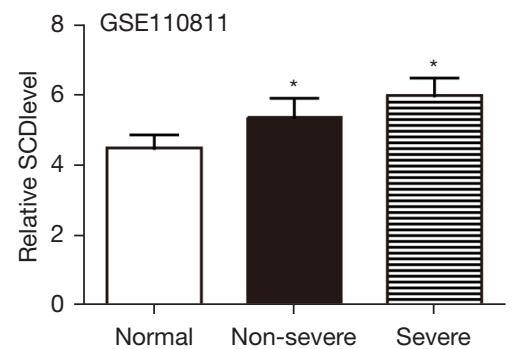

$\mathrm{F}$

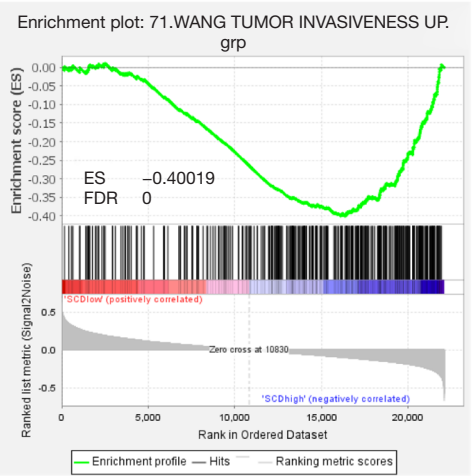

Figure 1 Network pharmacology analysis concluded that SCD may be one of the targets of ursolic acid, and the expression level of SCD is closely related to the clinical phenotype of Rb. (A) SwissTarget (top 15) and TargetNet (pro $>0$ ) were used to predict the targets of ursolic acid. GEO2R was used to analyze the up-regulated genes in the GSE97508, GSE24673, and GSE110811 datasets, then the intersection point with the ursolic acid target was obtained. (B) The expression level of SCD in the GSE97508, GSE24673, and GSE110811 datasets. (C) The correlation between SCD and disease severity in the GSE110811 dataset. (D-F) GSEA was used to analyze the relationship between SCD expression level and cell proliferation, migration, and invasion. *, $\mathrm{P}<0.05$. SCD, stearoyl-CoA desaturase; Rb, retinoblastoma; GSEA, gene set enrichment analysis.

\section{$U A$ inhibited the migration and invasion of $R b$ cells in vitro}

The results of the wound scratch test showed that UA inhibited SO-RB50 and Y79 cell migration compared with the $0 \mu \mathrm{M}$ UA group (Figure $3 A, 3 B$ ). The Transwell assay results showed that UA suppressed SO-RB50 and Y79 cell invasion compared with the $0 \mu M$ UA group (Figure 3C). These results suggested that $\mathrm{UA}$ inhibited $\mathrm{Rb}$ cell proliferation, migration, and invasion.

\section{UA suppressed the growth of transplanted tumors in vivo}

As shown in Figure 4A-4C, compared with the control group, UA suppressed the increase of transplanted tumor volume. UA also inhibited the increase of transplanted tumor weight (Figure 4D). These results suggested that UA suppressed the growth of transplanted tumors in vivo.

\section{$S C D$ promoted malignant phenotypes of $R b$ cells}

Western blot results showed that the relative protein expression level of SCD was significantly increased in transfected SO-RB50 and Y79 cells, indicating that the overexpression of SCD was successfully constructed (Figure 5A). The CCK-8 assay results showed that SCD overexpression promoted SO-RB50 and Y79 cell viability compared with the vector group (Figure 5B,5C). The results of the clone formation assay showed that the overexpression of SCD induced SO-RB50 and Y79 cell proliferation compared with the vector group (Figure 5D). The results of the wound scratch test showed that the overexpression of SCD promoted SO-RB50 and Y79 cell migration compared with the vector group (Figure 5E, 5F). Transwell assay results showed that SCD overexpression promoted SO-RB50 and Y79 cell invasion compared with the vector group (Figure $5 G$ ). 
A

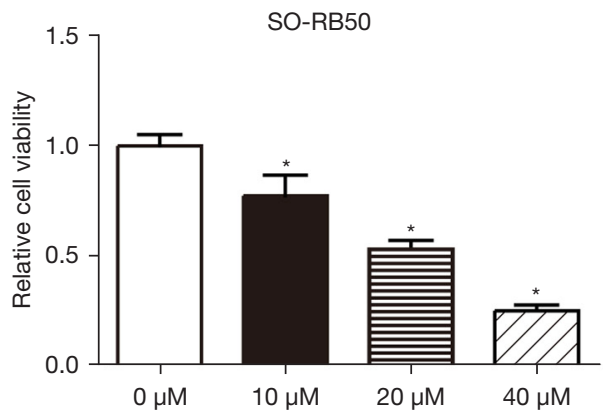

C

Ursolic acid 0

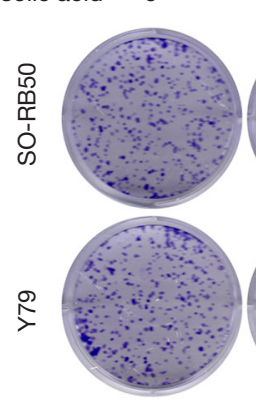

10

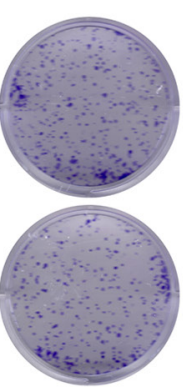

20

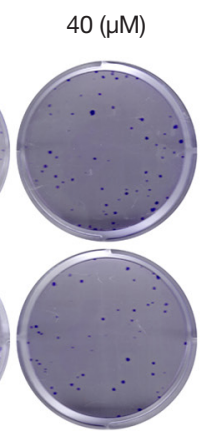

B

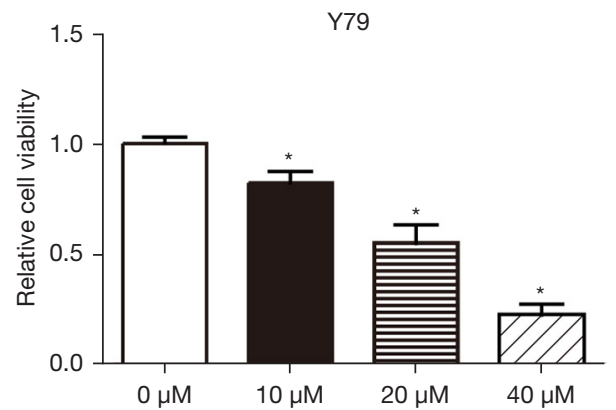

Figure 2 Ursolic acid inhibited cell proliferation in vitro. (A,B) The CCK-8 assay was used to assess SO-RB50 and Y79 cell viability. (C) The clone formation assay was used to assess SO-RB50 and Y79 cell proliferation (magnification 40x). Compared with the $0 \mu M$ group, *, $\mathrm{P}<0.05$. CCK, cell counting kit-8.

\section{Low SCD expression suppressed the proliferation, migration, and invasion of $R b$ cells in vitro}

Western blot was used to detect the relative protein expression level of SCD, and the results showed that the relative protein expression level of SCD was significantly decreased in transfected SO-RB50 and Y79 cells, indicating that low expression of SCD was successfully constructed (Figure 6A). The CCK-8 assay results showed that low SCD expression inhibited SO-RB50 and Y79 cell viability compared with the sh-NC group (Figure 6B,6C). The results of the clone formation assay showed that low expression of SCD suppressed SO-RB50 and Y79 cell proliferation compared with the sh-NC group (Figure $6 D$ ). The results of the wound scratch test showed that low expression of SCD inhibited SO-RB50 and Y79 cell migration compared with the sh-NC group (Figure 6E,6F). Transwell assay results showed that low SCD expression suppressed SO-RB50 and Y79 cell invasion compared with the sh-NC group (Figure 6G). These results suggested that $\mathrm{SCD}$ suppressed $\mathrm{Rb}$ cell proliferation, migration, and invasion.

\section{SCD inbibited inflammation as determined by GSEA and cytokine analysis}

According to the median SCD expression level, Rb patients were divided into 2 groups with high/low SCD expression, then GSEA was performed. The results showed that high expression levels of SCD inhibited inflammation (Figure 7A). RT-qPCR was used to detect the relative mRNA expression levels of IL-8, IL-6, CXCL1, and CCL2, and the results showed that the relative mRNA expression levels of IL-8, IL-6, CXCL1, and CCL2 were significantly decreased in both SO-RB50 and Y79 cells in the SCD group compared with the vector group (Figure $7 B, 7 C$ ). ELISA was used to detect the relative protein expression levels of IL- 8 and IL-6, and the results showed that the relative protein expression levels of IL-8 and IL-6 were significantly decreased in both SO-RB50 and Y79 cells in the SCD group compared with the vector group (Figure $7 D-7 G$ ). RT-qPCR was used to detect the relative mRNA expression levels of IL-8, IL-6, CXCL1, and CCL2, and the results showed that the relative mRNA expression levels of IL-8, IL-6, CXCL1, and CCL2 were significantly increased both in SO-RB50 and 
A
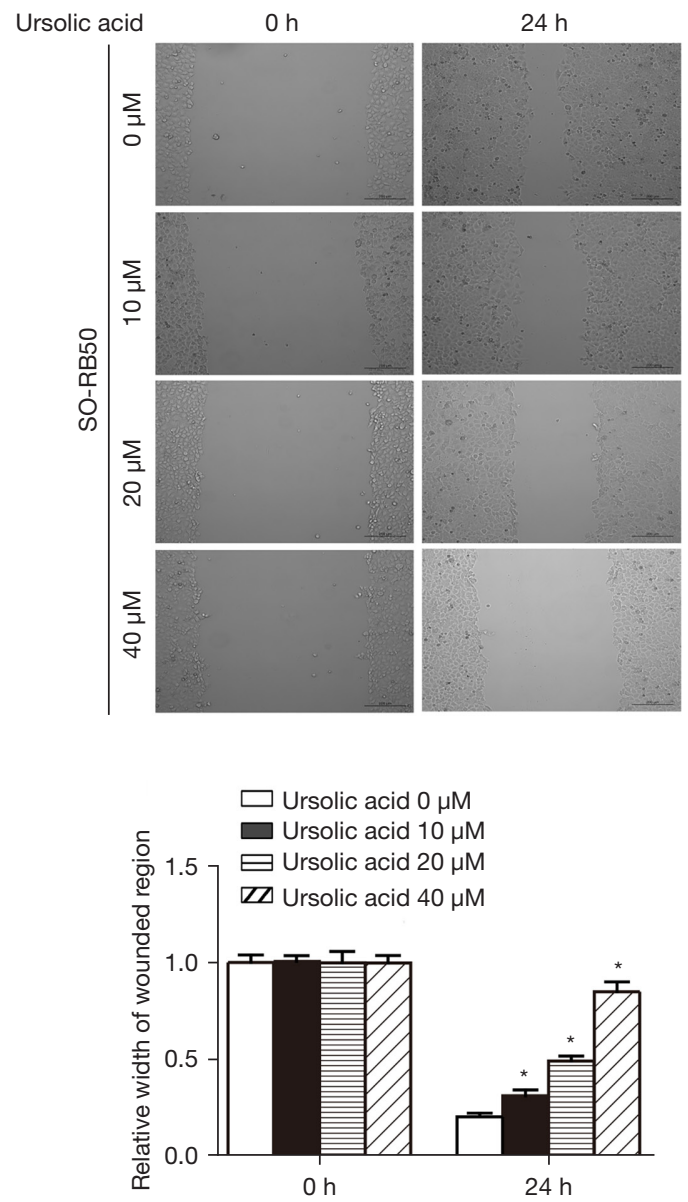

B

Ursolic acid $\mathrm{Oh}$
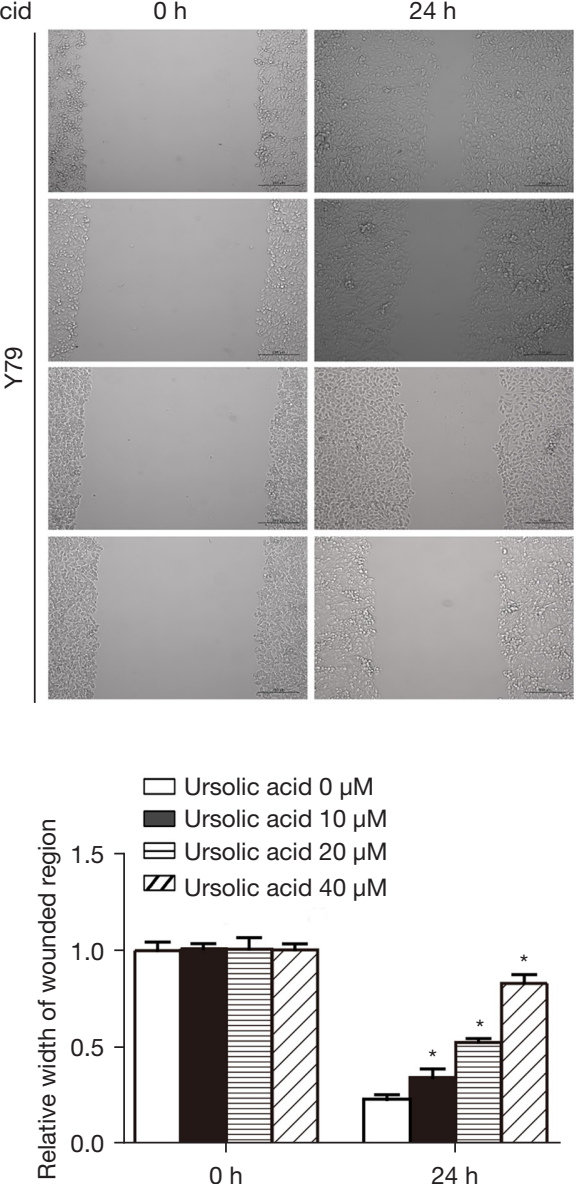

C
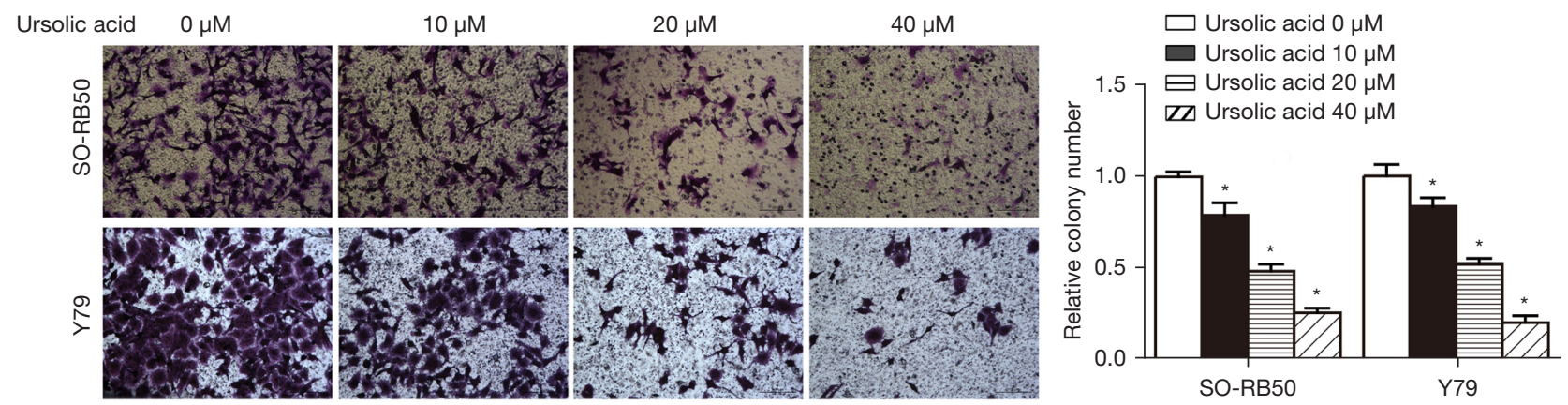

Figure 3 Ursolic acid inhibited the migration and invasion of $\mathrm{Rb}$ cells in vitro. (A,B) The wound scratch test was used to assess SO-RB50 and Y79 cell migration (magnification 100x). (C) The Transwell test was used to assess SO-RB50 and Y79 cell invasion compared with the $0 \mu \mathrm{M}$ ursolic acid group (magnification 200x). Compared with the $0 \mu \mathrm{M}$ group, ${ }^{*}, \mathrm{P}<0.05$.

Y79 cells in the shSCD 1\# and shSCD 2\# groups compared with the sh-NC group (Figure 7H,7I). ELISA was used to detect the relative protein expression levels of IL- 8 and
IL-6, and the results showed that the relative protein expression levels of IL-8 and IL-6 were significantly increased in both SO-RB50 and Y79 cells in the shSCD 1\# and shSCD 
A

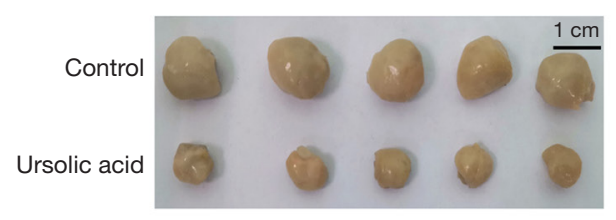

C

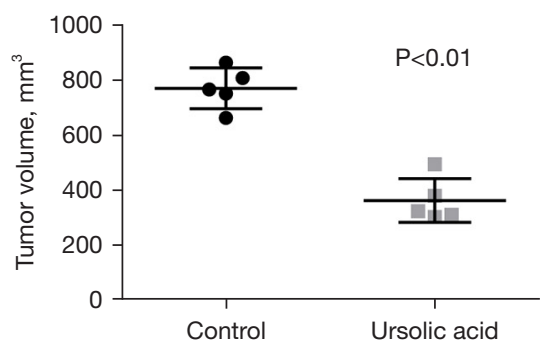

B

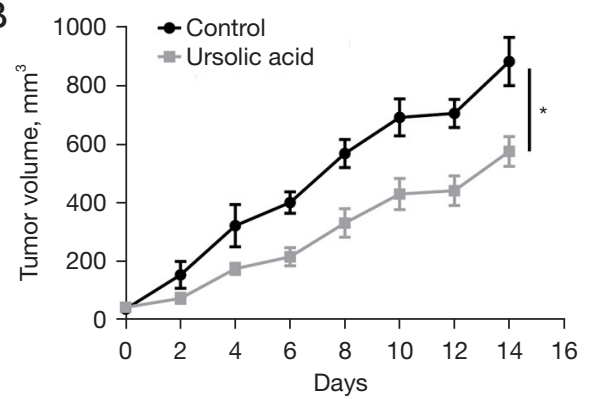

D

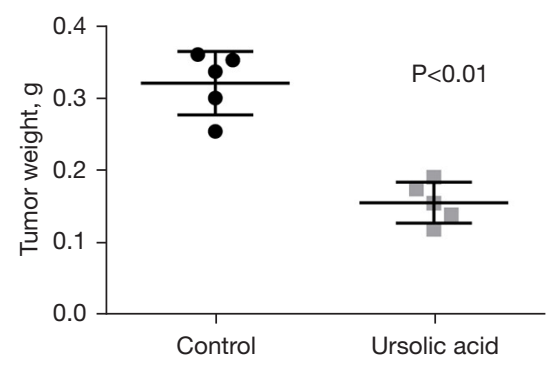

Figure 4 Ursolic acid suppressed the growth of transplanted tumors in vivo. (A-C) The transplanted tumor volumes were measured. (D) The transplanted weights were measured. Compared with the control group, ${ }^{*}, \mathrm{P}<0.05$.

2\# groups compared with the sh-NC group (Figure 7f-7N). These results suggested that SCD inhibited inflammation.

\section{UA inbibited the proliferation of Rb cells through SCD}

Western blot was used to detect the relative protein expression level of SCD, and the results showed that the relative protein expression level of SCD was significantly down-regulated in UA-treated SO-RB50 and Y79 cells. Furthermore, the relative protein expression level of SCD was down-regulated significantly with the increase of UA concentration, as the time of UA action increased (Figure 8A,8B). CCK-8 assay results showed that SO-RB50 and Y79 cell viability were inhibited in the UA + SCD group compared with the SCD group (Figure 8C, $8 D$ ). The results of the clone formation assay showed that SO-RB50 and Y79 cell proliferation were suppressed in the UA + SCD group compared with the SCD group (Figure 8E).

\section{UA inbibited the migration and invasion of Rb cells through SCD}

The results of the wound scratch test showed that SO-RB50 and Y79 cell migration were inhibited in the UA + SCD group compared with the SCD group (Figure 9A,9B). The Transwell assay results showed that SO-RB50 and Y79 cell invasion were suppressed in the UA + SCD group compared with the SCD group (Figure 9C). These results suggested that UA inhibited the proliferation, migration, and invasion of Rb cells through SCD.

\section{Discussion}

$\mathrm{Rb}$ mostly occurs in children under 5 years old and accounts for about $3 \%$ of all childhood tumors. It can affect one eye, both eyes, one after another or at the same time, and has a family genetic tendency. It is the most serious and harmful malignant tumor among infant eye diseases. Its visual prognosis is poor, and it is prone to intracranial and distant metastasis, which often endangers the life of the patient. The treatment method is mainly chemotherapy combined with local treatment. Chemotherapy mainly includes intravenous chemotherapy and arterial chemotherapy. The therapeutic effect is limited and the side effects are relatively large. In recent years, with the continuous advancement of clinical diagnosis and treatment methods, the treatment of $\mathrm{Rb}$ has been greatly improved, but early detection is still difficult. Overall, finding ways to inhibit the proliferation, migration, and invasion of $\mathrm{Rb}$ will facilitate great progress in the diagnosis and treatment of $\mathrm{Rb}$.

$\mathrm{UA}$ is a five-ring triterpenoid compound with a wide range of pharmacological activities. Its anti-tumor effect 
A

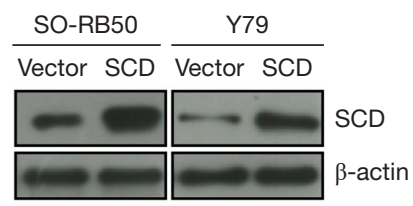

D

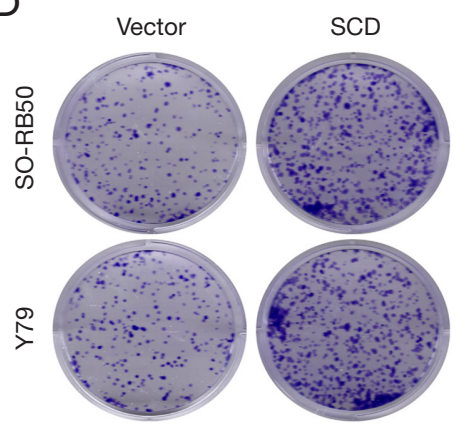

E

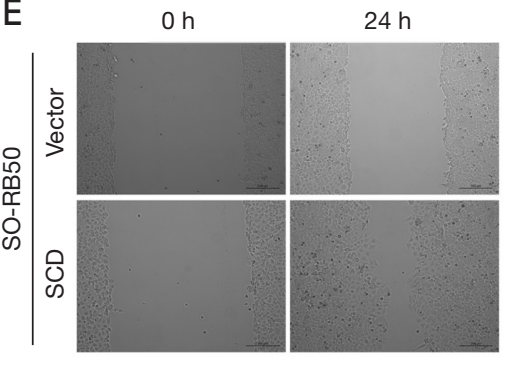

$\mathrm{F}$

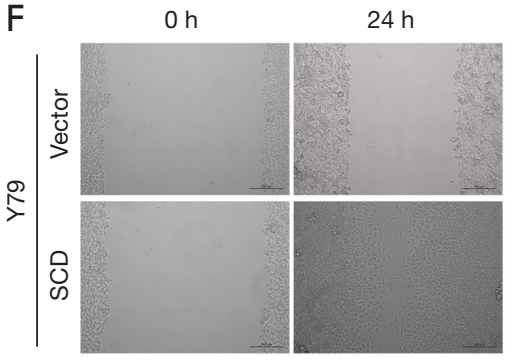

B
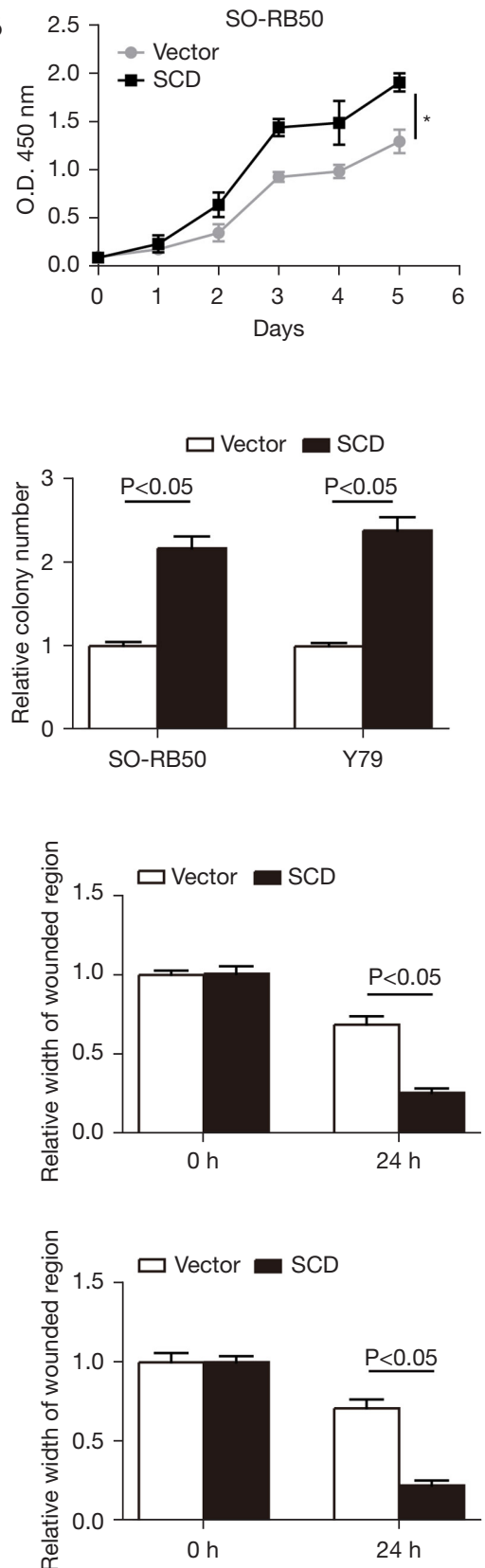

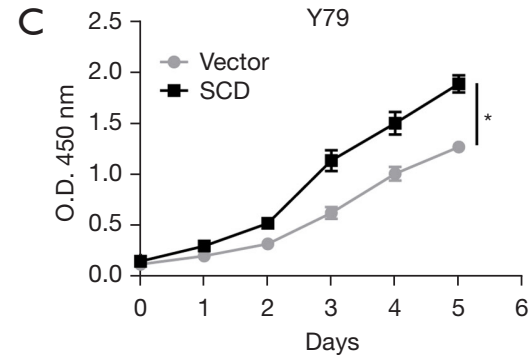

G
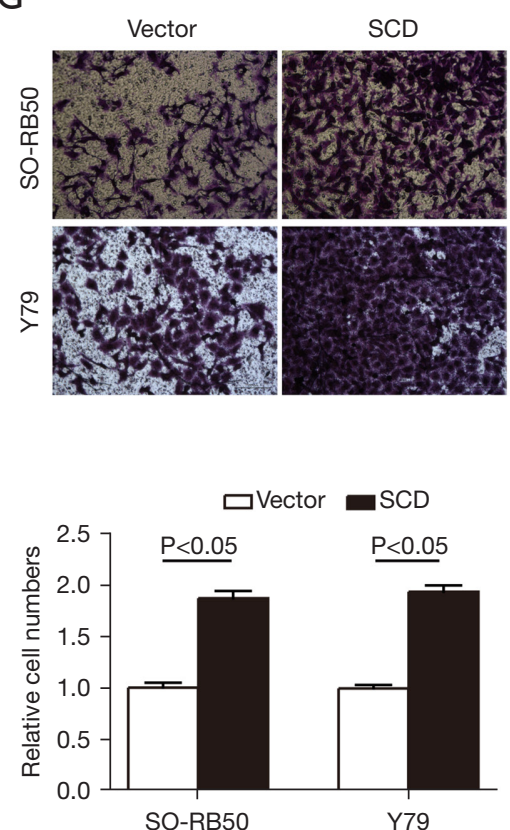

Figure 5 SCD promoted the proliferation, migration, and invasion of $\mathrm{Rb}$ cells in vitro. (A) Western blot was used to detect the relative protein expression level of SCD. (B,C) The CCK-8 assay was used to assess SO-RB50 and Y79 cell viability. (D) The clone formation assay was used to assess SO-RB50 and Y79 cell proliferation (magnification 40x). (E,F) The wound scratch test was used to assess SO-RB50 and Y79 cell migration (magnification 100x). (G) The Transwell assay was used to assess SO-RB50 and Y79 cell invasion (magnification 200x). Compared with the vector group, ${ }^{*}, \mathrm{P}<0.05$. SCD, stearoyl-CoA desaturase.

is particularly prominent. The anti-tumor effect of UA has been reported in a variety of diseases, including breast cancer (31), leukemia (32), prostate cancer (33), melanoma (34), endometrial cancer (35), and lung cancer (36), among others. A large number of studies have shown that UA can inhibit tumor growth and metastasis by inhibiting tumor cell proliferation, invasion, and metastasis, and its mechanism is related to the expression of related genes on tumor cells, 
A

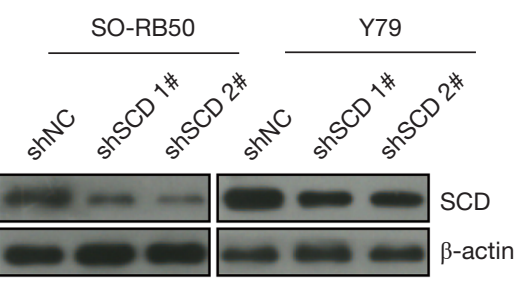

D

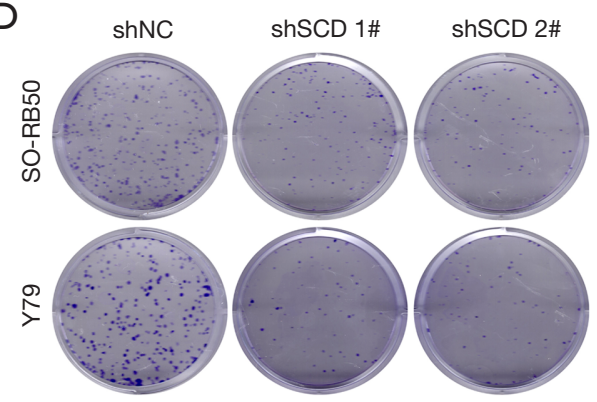

E
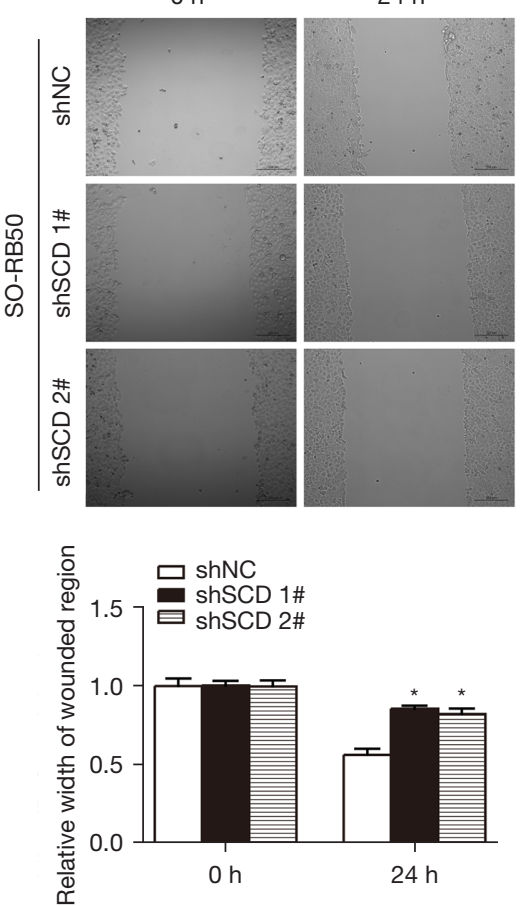

B
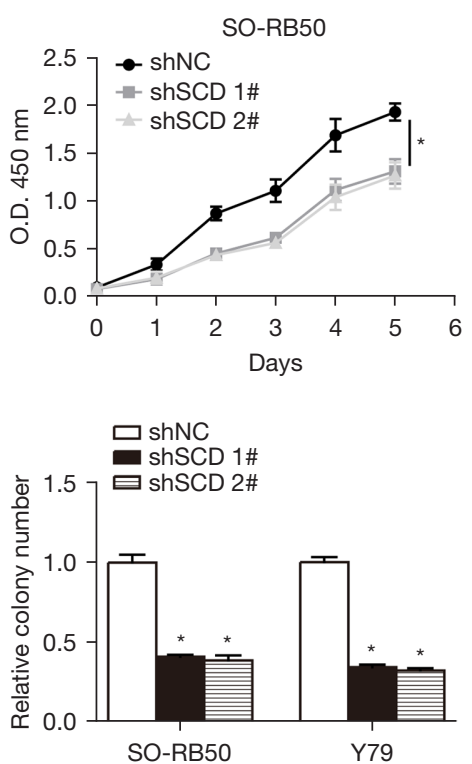

$\mathrm{F}$
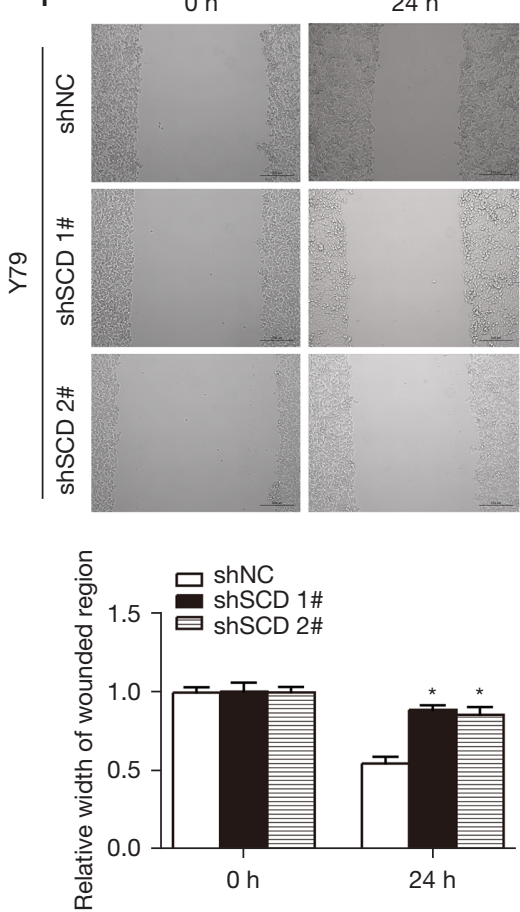

C

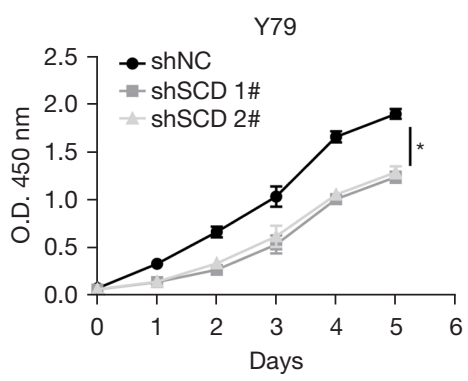

G
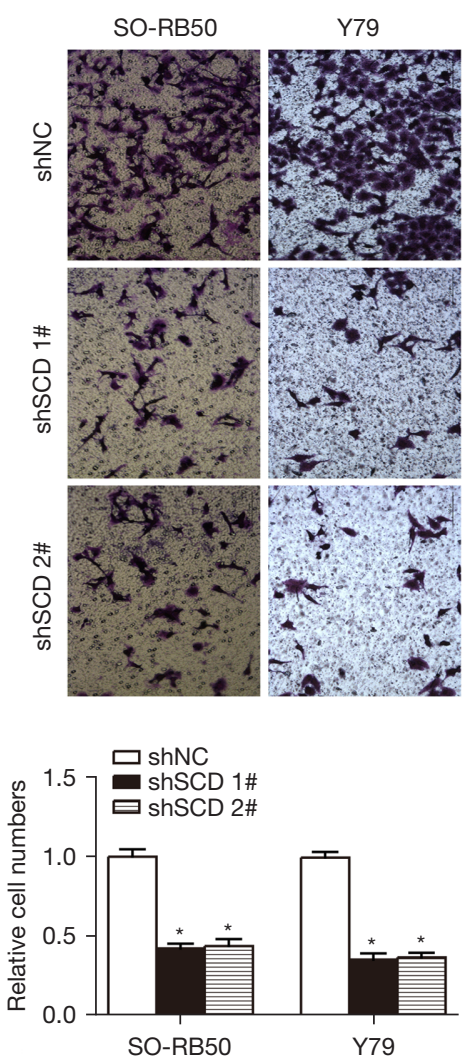

Figure 6 Low SCD expression inhibited the proliferation, migration, and invasion of Rb cells in vitro. (A) Western blot was used to detect the relative protein expression level of SCD. (B,C) The CCK-8 assay was used to assess SO-RB50 and Y79 cell viability. (D) The clone formation assay was used to assess SO-RB50 and Y79 cell proliferation (magnification 40×). (E,F) The wound scratch test was used to assess SO-RB50 and Y79 cell migration (magnification 100x). (G) The Transwell assay was used to assess SO-RB50 and Y79 cell invasion (magnification 200x). Compared with the sh-NC group, *, $\mathrm{P}<0.05$. SCD, stearoyl-CoA desaturase; Rb, retinoblastoma; CCK, cell counting kit-8. 
A

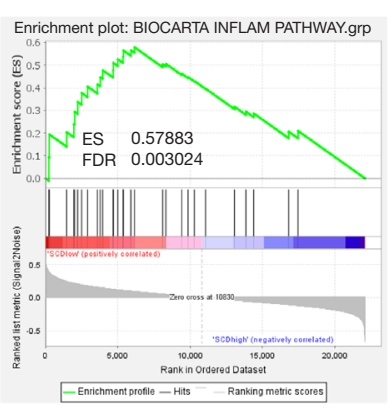

D

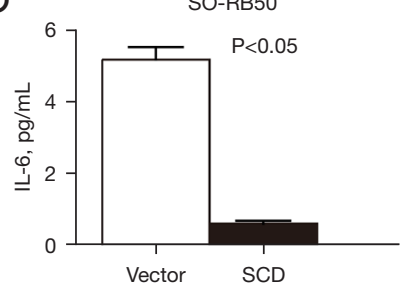

$\mathrm{F}$

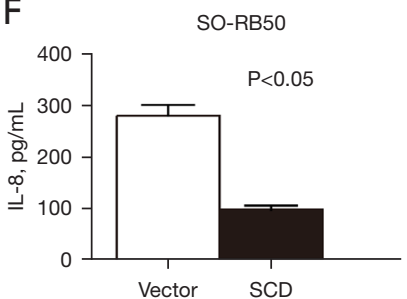

$\mathrm{H}$
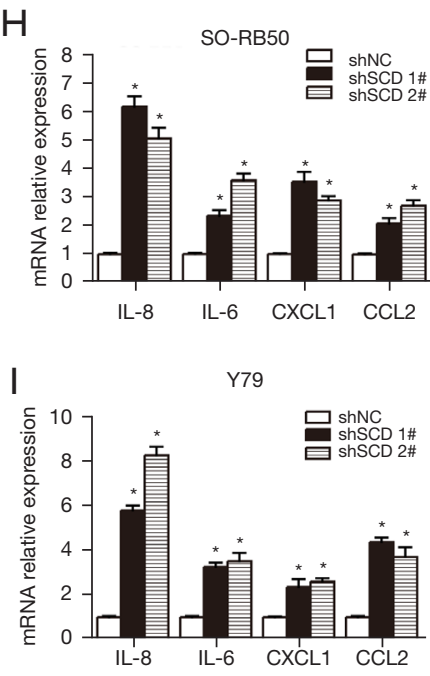

B

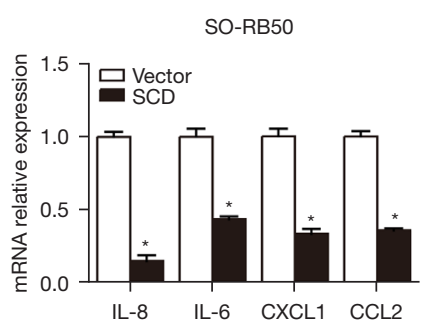

$E$

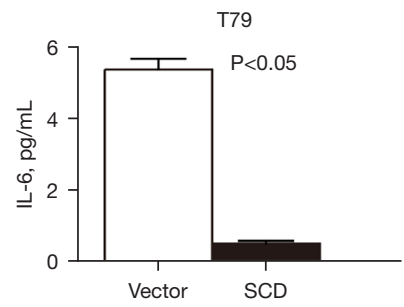

G

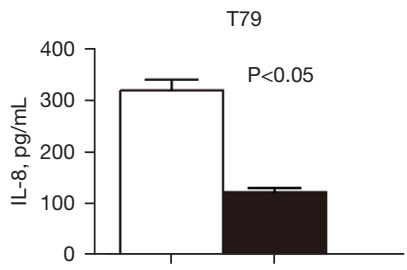

J

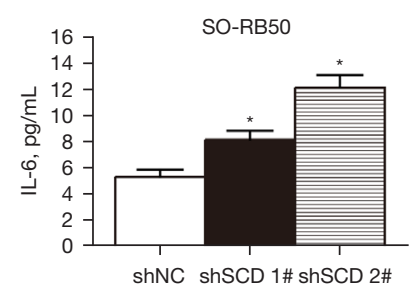

$\mathrm{L}$

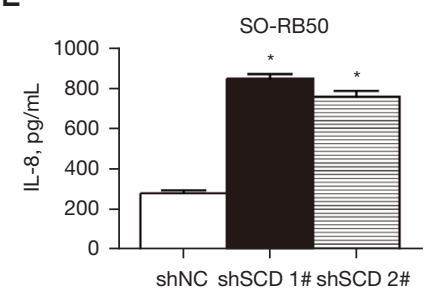

C

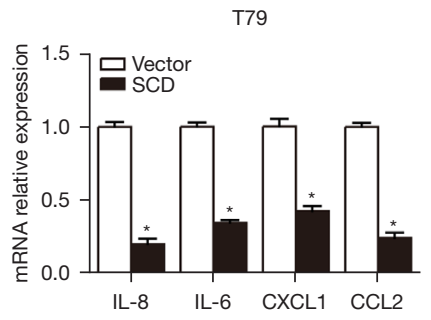

K

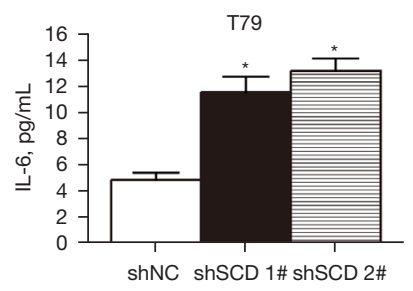

M

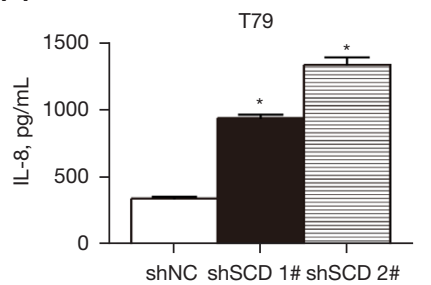

Figure 7 SCD inhibited inflammation as determined by GSEA and cytokine analysis. (A) GSEA was used to analyze the relationship between SCD expression level and inflammation. (B,C) RT-qPCR was used to detect the relative mRNA expression levels of IL-8, IL-6, CXCL1, and CCL2 in SO-RB50 and Y79 cells. (D-G) ELISA was used to detect the relative protein expression levels of IL-8 and IL-6 in SO-RB50 and Y79 cells. (H,I) RT-qPCR was used to detect the relative mRNA expression levels of IL-8, IL-6, CXCL1, and CCL2 in SORB50 and Y79 cells transfected with shRNA NC, shRNA SCD 1\#, or shRNA SCD 2\#. (J-N) ELISA was used to detect the relative protein expression levels of IL-8 and IL-6 in SO-RB50 and Y79 cells. Compared with the vector group, ${ }^{*}, \mathrm{P}<0.05$; compared with the sh-NC group, *, $\mathrm{P}<0.05$. SCD, stearoyl-CoA desaturase; GSEA, gene set enrichment analysis. 
A

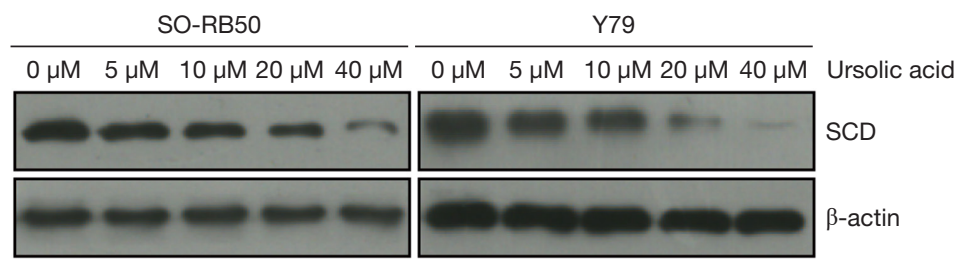

B

\begin{tabular}{|c|c|c|c|c|c|c|c|c|}
\hline \multicolumn{4}{|c|}{ SO-RB50 } & \multicolumn{4}{|c|}{ Y79 } & \\
\hline+ & + & + & + & + & + & + & + & Ursolic acid \\
\hline 0 & 12 & 24 & 48 & 0 & 12 & 24 & 48 & Time, h \\
\hline & & & $-\infty$ & & & $=$ & $=$ & SCD \\
\hline & & & & & & & & $\beta$-actin \\
\hline
\end{tabular}

\section{$\mathrm{E}$}
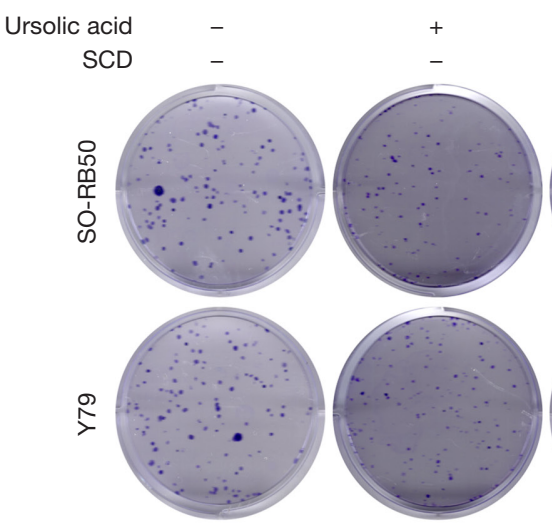

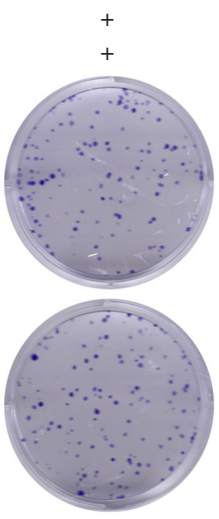

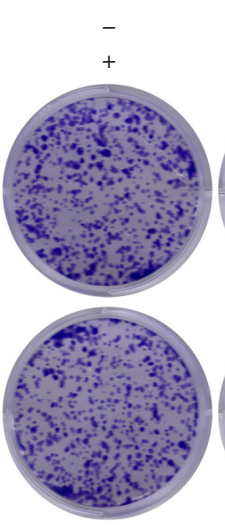

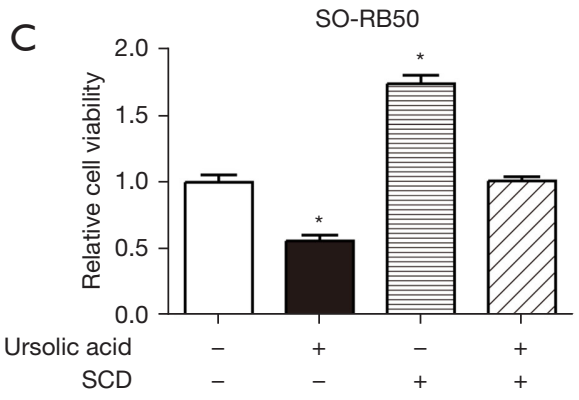

D

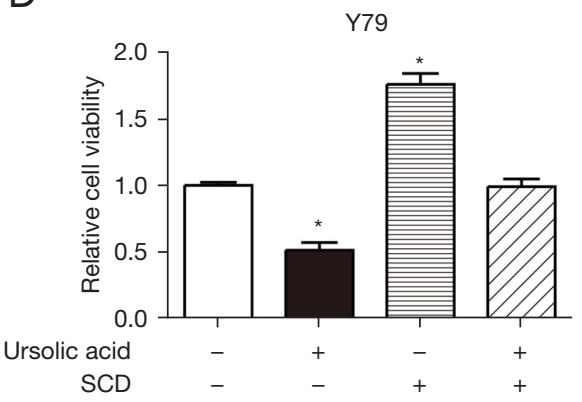

Figure 8 Ursolic acid inhibited the proliferation of Rb cells through SCD. (A,B) Western blot was used to detect the relative protein expression level of SCD. (C,D) The CCK-8 assay was used to assess SO-RB50 and Y79 cell viability. (E) The clone formation assay was used to assess SO-RB50 and Y79 cell proliferation (magnification 40×). Compared with the control + vector group, ${ }^{*}, \mathrm{P}<0.05$. Rb, retinoblastoma; SCD, stearoyl-CoA desaturase; CCK, cell counting kit-8.

signal transduction pathways of tumor cells, and the cell cycle of tumor cells (37). Silva et al. showed that UA can inhibit the proliferation of $\mathrm{Rb}$ cells and promote their apoptosis. It is therefore one of the most promising anti-Rb drugs (17). In this study, UA inhibited $\mathrm{Rb}$ cell proliferation, migration, and invasion. At the same time, UA suppressed the growth of transplanted tumors in vivo.

SCD has 2 functions in the body, namely $\beta$-oxidation of fatty acids and the regulation of lipid synthesis. Normally, fatty acids enter the mitochondria and are oxidized inside the mitochondria. The rate-limiting step of this process relies on the mitochondrial carnitine palmitoyltransferase shuttle system, which cannot function without malonylCoA. When the expression of SCD1 decreases, it will lead to an increase in SFA. The increase in SFA can directly inhibit the activity of acetyl-CoA carboxylase (ACC), thereby reducing the level of malonyl-CoA in the cell. Therefore, as the expression of SCD1 decreases, 
A
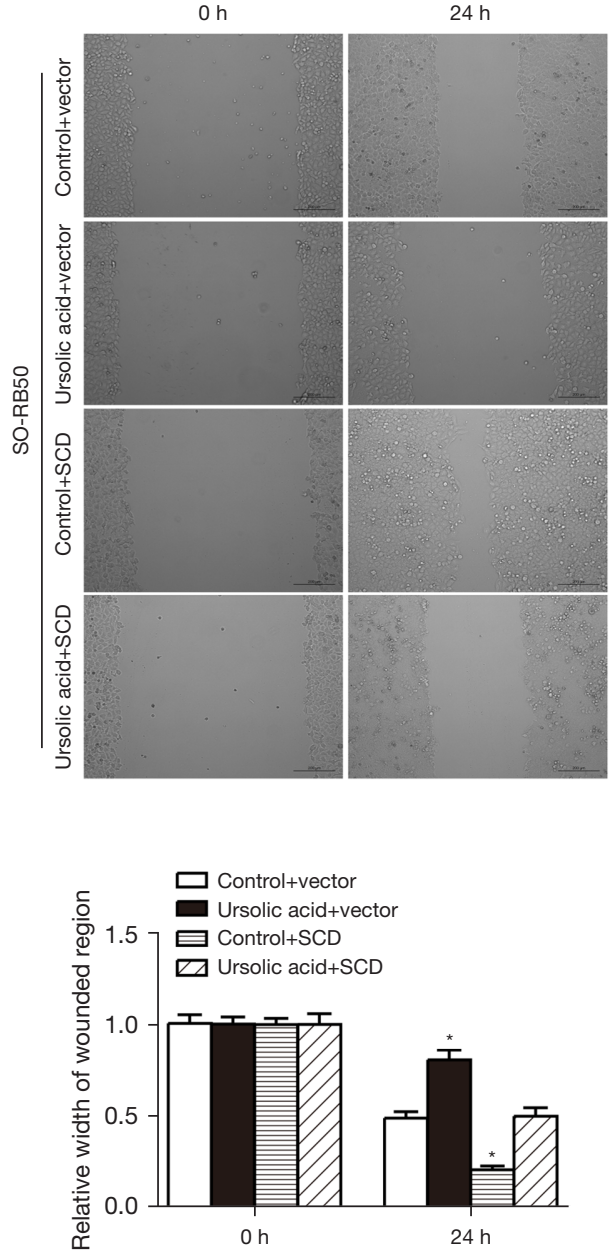

B
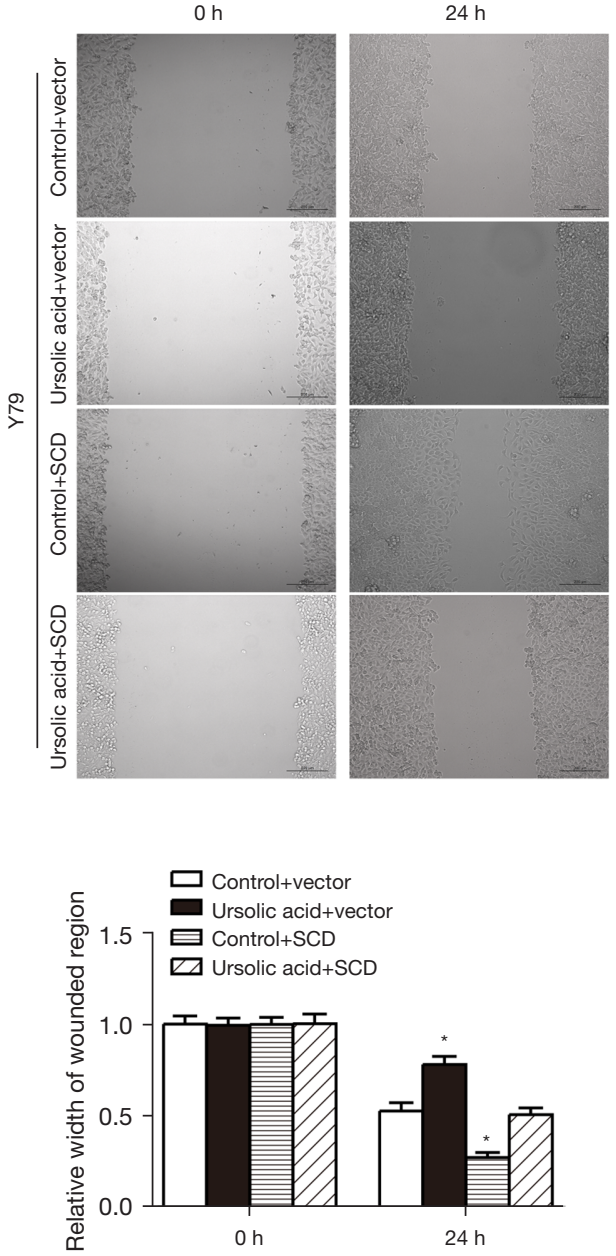

C
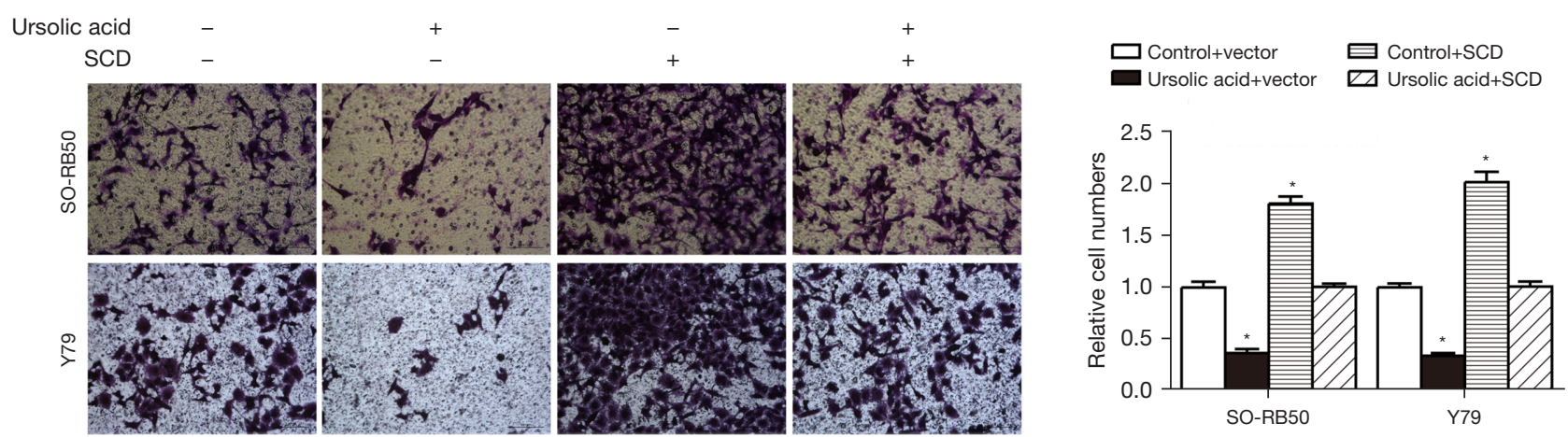

Figure 9 Ursolic acid inhibited the proliferation, migration, and invasion of Rb cells through SCD. (A,B) The wound scratch test was used to assess SO-RB50 and Y79 cell migration (magnification 100x). (C) The Transwell assay was used to assess SO-RB50 and Y79 cell invasion (magnification 200×). Compared with the control + vector group, ${ }^{*}, \mathrm{P}<0.05$. Rb, retinoblastoma; SCD, stearoyl-CoA desaturase. 
the oxidation of fatty acids increases, and the ability to synthesize fatty acids decreases. On the one hand, the energy supply required by tumor tissues decreases. On the other hand, the formation of cell membranes is inhibited and the ability to divide rapidly is weakened (38). With the deepening of the understanding of the SCD gene, SCD has been shown to be involved in the occurrence and development of a variety of malignant tumors. Many studies have pointed out that SCD is highly expressed in malignant tumors such as lung cancer, gastrointestinal tumors, urinary system tumors, and gynecological tumors. Reducing the expression of SCD at the genetic or pharmacological level can inhibit the proliferation, invasion, and migration of many different types of tumor cells (39). In this study, overexpression of SCD promoted the proliferation, migration, and invasion of $\mathrm{Rb}$ cells in vitro, while low expression of SCD inhibited the proliferation, migration, and invasion of Rb cells in vitro. Therefore, SCD inhibition may be a novel target for anticancer therapy $(39,40)$. Furthermore, we observed that the expression level of SCD was significantly down-regulated in SO-RB50 and Y79 cells treated with UA, which proved the inhibitory effect of UA on SCD. Also, UA inhibited the facilitation of SCD on the cell proliferation, migration, and invasion of SO-RB50 and Y79 cells.

A large number of studies have proven the important role of SCD expression in lipid metabolism. This enzyme is not only involved in the pathology of obesity, but is also a key regulator of other types of serious diseases related to inflammation and stress. The expression of SCD needs to be strictly controlled within the normal range to maintain cell function. The sharp increase in SCD expression is conducive to fat accumulation, leading to obesity and insulin resistance, while significant suppression of SCD expression may promote fat catabolism, but at the cost of increased cell inflammation and stress. Therefore, the dysfunction of SCD expression regulation is associated with many types of serious diseases, such as obesity, insulin resistance, cancer, and inflammatory diseases. Although SCD deficiency provides beneficial metabolic effects, it actually causes pancreatic $\beta$-cell dysfunction in vitro and in vivo. Observations of the effects of SCD deficiency on liver stress and inflammatory diseases (including atherosclerosis and enterocolitis) also vary in different studies, which may be due to the different disease models used and the methods used to inhibit SCD expression (41). The latest research showed that the absence of SCD in melanoma promotes inflammation (42). In this study, GSEA showed that high expression levels of SCD inhibited inflammation. At the same time, cytokine analysis results showed that overexpression of SCD inhibited inflammation, while low expression of SCD promoted inflammation.

In summary, UA can inhibit the proliferation, migration, and invasion of $\mathrm{Rb} \mathrm{SO}-\mathrm{RB} 50$ and $\mathrm{Y} 79$ cells, and its mechanism of action may be achieved by down-regulating the expression of SCD. This study thoroughly explored the related molecular regulatory mechanisms of UA in inhibiting the proliferation, migration, and invasion of $\mathrm{Rb}$ SO-RB50 and Y79 cells, which provides a theoretical basis for further basic research, clinical research, and applications of UA in the future. It can be expected that with the indepth research and clinical promotion of the anti-tumor mechanism of UA, it may become a natural anti-tumor drug with few side effects, good efficacy, and a low price.

\section{Acknowledgments}

Funding: None.

\section{Footnote}

Reporting Checklist: The authors have completed the ARRIVE reporting checklist. Available at https://dx.doi. org/10.21037/atm-21-4617

Data Sharing Statement: Available at https://dx.doi. org/10.21037/atm-21-4617

Conflicts of Interest: All authors have completed the ICMJE uniform disclosure form (available at https://dx.doi. org/10.21037/atm-21-4617). The authors have no conflicts of interest to declare.

Etbical Statement: The authors are accountable for all aspects of the work in ensuring that questions related to the accuracy or integrity of any part of the work are appropriately investigated and resolved. The study was conducted in accordance with the Declaration of Helsinki (as revised in 2013). All animal experiments were carried out according to the NIH Guide for the Care and Use of Laboratory Animals and were approved by the Suzhou high tech Zone People's Hospital.

Open Access Statement: This is an Open Access article distributed in accordance with the Creative Commons Attribution-NonCommercial-NoDerivs 4.0 International 
License (CC BY-NC-ND 4.0), which permits the noncommercial replication and distribution of the article with the strict proviso that no changes or edits are made and the original work is properly cited (including links to both the formal publication through the relevant DOI and the license). See: https://creativecommons.org/licenses/by-nc-nd/4.0/.

\section{References}

1. Singh L, Kashyap S. Update on pathology of retinoblastoma. Int J Ophthalmol 2018;11:2011-6.

2. Knudson AG Jr. Mutation and cancer: statistical study of retinoblastoma. Proc Natl Acad Sci U S A 1971;68:820-3.

3. Broaddus E, Topham A, Singh AD. Incidence of retinoblastoma in the USA: 1975-2004. Br J Ophthalmol 2009;93:21-3.

4. Francis JH, Levin AM, Abramson DH. Update on Ophthalmic Oncology 2014: Retinoblastoma and Uveal Melanoma. Asia Pac J Ophthalmol (Phila) 2016;5:368-82.

5. Kivelä $T$. The epidemiological challenge of the most frequent eye cancer: retinoblastoma, an issue of birth and death. Br J Ophthalmol 2009;93:1129-31.

6. Naseripour M, Nazari H, Bakhtiari P, et al. Retinoblastoma in Iran: outcomes in terms of patients' survival and globe survival. Br J Ophthalmol 2009;93:28-32.

7. Seregard S, Lundell G, Svedberg H, et al. Incidence of retinoblastoma from 1958 to 1998 in Northern Europe: advantages of birth cohort analysis. Ophthalmology 2004;111:1228-32.

8. Chantada GL, Qaddoumi I, Canturk S, et al. Strategies to manage retinoblastoma in developing countries. Pediatr Blood Cancer 2011;56:341-8.

9. Canturk S, Qaddoumi I, Khetan V, et al. Survival of retinoblastoma in less-developed countries impact of socioeconomic and health-related indicators. $\mathrm{Br} \mathrm{J}$ Ophthalmol 2010;94:1432-6.

10. Golabchi K, Soleimani-Jelodar R, Aghadoost N, et al. MicroRNAs in retinoblastoma: Potential diagnostic and therapeutic biomarkers. J Cell Physiol 2018;233:3016-23.

11. Wan SZ, Liu C, Huang CK, et al. Ursolic Acid Improves Intestinal Damage and Bacterial Dysbiosis in Liver Fibrosis Mice. Front Pharmacol 2019;10:1321.

12. Cheng J, Liu Y, Liu Y, et al. Ursolic acid alleviates lipid accumulation by activating the AMPK signaling pathway in vivo and in vitro. J Food Sci 2020;85:3998-4008.

13. Zhou M, Yi Y, Liu L, et al. Polymeric micelles loading with ursolic acid enhancing anti-tumor effect on hepatocellular carcinoma. J Cancer 2019;10:5820-31.
14. González-Garibay AS, López-Vázquez A, García-Bañuelos $\mathrm{J}$, et al. Effect of Ursolic Acid on Insulin Resistance and Hyperinsulinemia in Rats with Diet-Induced Obesity: Role of Adipokines Expression. J Med Food 2020;23:297-304.

15. Woźniak Ł, Skąpska S, Marszałek K. Ursolic Acid-A Pentacyclic Triterpenoid with a Wide Spectrum of Pharmacological Act ivities. Molecules 2015;20:20614-41.

16. Huang CY, Lin CY, Tsai CW, et al. Inhibition of cell proliferation, invasion and migration by ursolic acid in human lung cancer cell lines. Toxicol In Vitro 2011;25:1274-80.

17. Silva AM, Alvarado HL, Abrego G, et al. In Vitro Cytotoxicity of Oleanolic/Ursolic Acids-Loaded in PLGA Nanoparticles in Different Cell Lines. Pharmaceutics 2019;11:362.

18. Heinemann FS, Ozols J. Stearoyl-CoA desaturase, a shortlived protein of endoplasmic reticulum with multiple control mechanisms. Prostaglandins Leukot Essent Fatty Acids 2003;68:123-33.

19. Jeffcoat R, Brawn PR, Safford R, et al. Properties of rat liver microsomal stearoyl-coenzyme A desaturase. Biochem J 1977;161:431-7.

20. Igal RA. Stearoyl-CoA desaturase-1: a novel key player in the mechanisms of cell proliferation, programmed cell death and transformation to cancer. Carcinogenesis 2010;31:1509-15.

21. Nashed M, Chisholm JW, Igal RA. Stearoyl-CoA desaturase activity modulates the activation of epidermal growth factor receptor in human lung cancer cells. Exp Biol Med (Maywood) 2012;237:1007-17.

22. von Roemeling CA, Marlow LA, Wei JJ, et al. StearoylCoA desaturase 1 is a novel molecular therapeutic target for clear cell renal cell carcinoma. Clin Cancer Res 2013;19:2368-80.

23. Vargas T, Moreno-Rubio J, Herranz J, et al. ColoLipidGene: signature of lipid metabolism-related genes to predict prognosis in stage-II colon cancer patients. Oncotarget 2015;6:7348-63.

24. Fritz V, Benfodda Z, Rodier G, et al. Abrogation of de novo lipogenesis by stearoyl-CoA desaturase 1 inhibition interferes with oncogenic signaling and blocks prostate cancer progression in mice. Mol Cancer Ther 2010;9:1740-54.

25. Keller A, Leidinger P, Bauer A, et al. Toward the bloodborne miRNome of human diseases. Nat Methods 2011;8:841-3.

26. Keller A, Leidinger P, Vogel B, et al. miRNAs can be generally associated with human pathologies as exemplified 


\section{Page 16 of 16}

for miR-144. BMC Med 2014;12:224.

27. Kong L, Liu P, Zheng M, et al. The miR-1224-5p/ELF3 Axis Regulates Malignant Behaviors of Pancreatic Cancer via PI3K/AKT/Notch Signaling Pathways. Onco Targets Ther 2020;13:3449-66.

28. Subramanian A, Tamayo P, Mootha VK, et al. Gene set enrichment analysis: a knowledge-based approach for interpreting genome-wide expression profiles. Proc Natl Acad Sci U S A 2005;102:15545-50.

29. Mickey DD, Stone KR, Wunderli H, et al. Heterotransplantation of a human prostatic adenocarcinoma cell line in nude mice. Cancer Res 1977;37:4049-58.

30. Shanmugam MK, Rajendran P, Li F, et al. Ursolic acid inhibits multiple cell survival pathways leading to suppression of growth of prostate cancer xenograft in nude mice. J Mol Med (Berl) 2011;89:713-27.

31. Xiang F, Fan Y, Ni Z, et al. Ursolic Acid Reverses the Chemoresistance of Breast Cancer Cells to Paclitaxel by Targeting MiRNA-149-5p/MyD88. Front Oncol 2019;9:501.

32. Ma D, Fang Q, Li Y, et al. Crucial role of heme oxygenase- 1 in the sensitivity of acute myeloid leukemia cell line Kasumi-1 to ursolic acid. Anticancer Drugs 2014;25:406-14.

33. Mu D, Zhou G, Li J, et al. Ursolic acid activates the apoptosis of prostate cancer via ROCK/PTEN mediated mitochondrial translocation of cofilin-1. Oncol Lett 2018;15:3202-6

34. AlQathama A, Shao L, Bader A, et al. Differential AntiProliferative and Anti-Migratory Activities of Ursolic Acid, 3-O-Acetylursolic Acid and Their Combination

Cite this article as: Zhou D, Bao Q, Fu S. Anticancer activity of ursolic acid on retinoblastoma cells determined by bioinformatics analysis and validation. Ann Transl Med 2021;9(20):1548. doi: 10.21037/atm-21-4617
Zhou et al. Bioinformatics study of Ursolic acid in retinoblastoma

Treatments with Quercetin on Melanoma Cells.

Biomolecules 2020;10:894.

35. Achiwa Y, Hasegawa K, Udagawa Y. Effect of ursolic acid on MAPK in cyclin D1 signaling and RING-type E3 ligase (SCF E3s) in two endometrial cancer cell lines. Nutr Cancer 2013;65:1026-33.

36. Gou W, Luo N, Wei H, et al. Ursolic acid derivative UA232 evokes apoptosis of lung cancer cells induced by endoplasmic reticulum stress. Pharm Biol 2020;58:707-15.

37. Baishya R, Nayak DK, Kumar D, et al. Ursolic Acid Loaded PLGA Nanoparticles: in vitro and in vivo Evaluation to Explore Tumor Targeting Ability on B16F10 Melanoma Cell Lines. Pharm Res 2016;33:2691-703.

38. She K, Fang S, Du W, et al. SCD1 is required for EGFRtargeting cancer therapy of lung cancer via re-activation of EGFR/PI3K/AKT signals. Cancer Cell Int 2019;19:103.

39. Ascenzi F, De Vitis C, Maugeri-Saccà M, et al. SCD1, autophagy and cancer: implications for therapy. J Exp Clin Cancer Res 2021;40:265.

40. Piao C, Cui X, Zhan B, et al. Inhibition of stearoyl CoA desaturase- 1 activity suppresses tumour progression and improves prognosis in human bladder cancer. J Cell Mol Med 2019;23:2064-76.

41. Liu X, Strable MS, Ntambi JM. Stearoyl CoA desaturase 1: role in cellular inflammation and stress. Adv Nutr 2011;2:15-22.

42. Vivas-García Y, Falletta P, Liebing J, et al. LineageRestricted Regulation of SCD and Fatty Acid Saturation by MITF Controls Melanoma Phenotypic Plasticity. Mol Cell 2020;77:120-137.e9.

(English Language Editor: C. Betlazar-Maseh) 